\title{
Berichte zur Lebensmittelsicherheit 2005
}

Nationaler Rückstandskontrollplan für Lebensmittel tierischen Ursprungs Inspektionsbericht Bericht zum Schnellwarnsystem Bericht zur Futtermittelüberwachung

Gemeinsamer Bericht des Bundes und der Länder 


\section{Inhaltsverzeichnis}

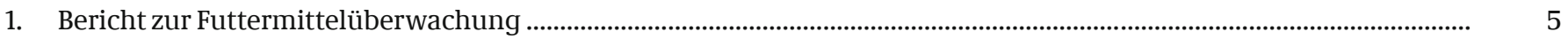

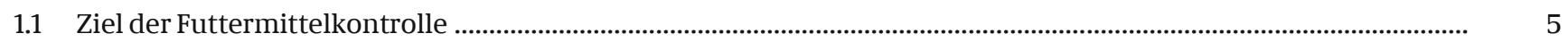

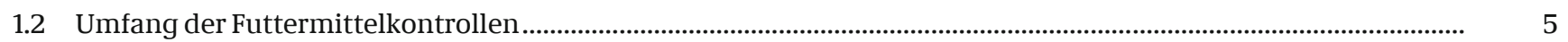

1.3 Anzahl der Probennahmen und der Einzelbestimmungen ...................................................................................... 6

1.4 Anzahl der Einzelbestimmungen auf Inhaltsstoffe und Energie .................................................................................

1.5 Anzahl der Einzelbestimmungen auf Futtermittel-Zusatzsstoffe.............................................................................

1.6 Anzahl der Einzelbestimmungen auf unzulässige Stoffe .......................................................................................

1.7 Anzahl der Einzelbestimmungen auf unerwünschte Stoffe (einschließlich Schädlingsbekämpfungsmittel) ........... 9

1.8 Anzahl der Einzelbestimmungen auf verbotene Stoffe ..............................................................................................

1.9 Kontrolle der Zusammensetzung von Mischfuttermitteln .......................................................................................

1.10 Anzahl der Untersuchungen auf mikrobiellen Verderb ............................................................................................

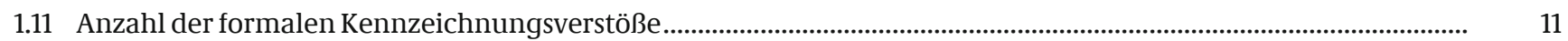

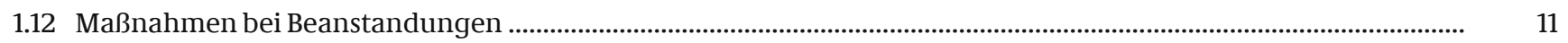

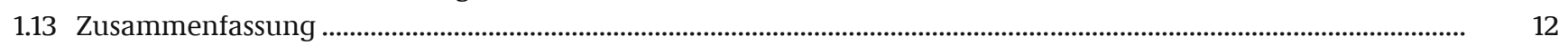

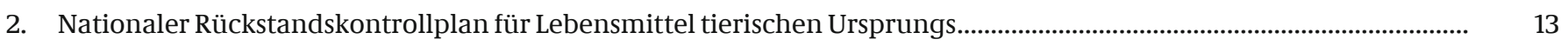

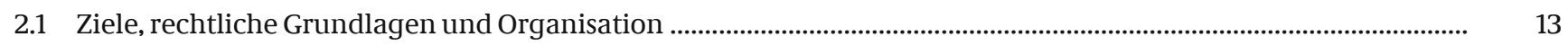

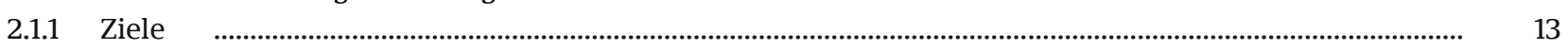

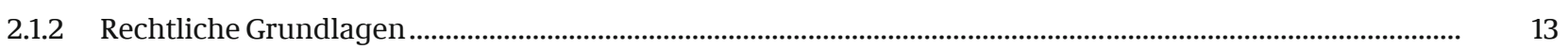

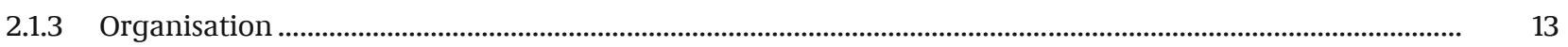

2.1.4 Untersuchungen ....................................................................................................................................

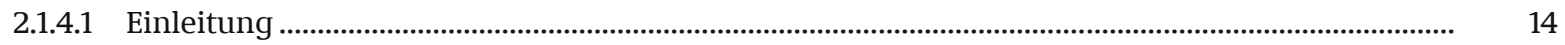

2.1.4.2 Stoffgruppen nach Anhang I der Richtlinie 96/23/EG .......................................................................... 15

2.1.4.3 Untersuchungshäufigkeit................................................................................................................................ 20

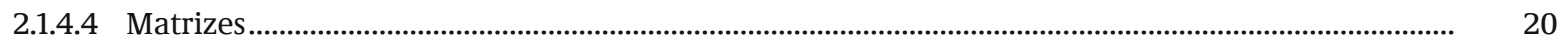

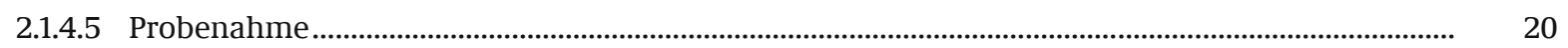

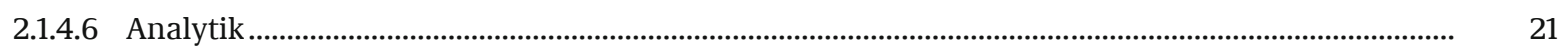

2.1.5 Maßnahmen für Tiere oder Erzeugnisse, bei denen Rückstände festgestellt wurden....................................... 21

2.2. Überblick über die Rückstandsuntersuchungen im Jahr 2005 .................................................................................. 22

2.3. Positive Rückstandsbefunde im Einzelnen.............................................................................................................. 22

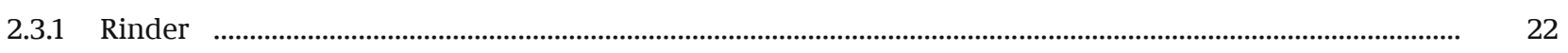

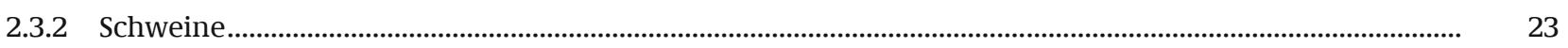

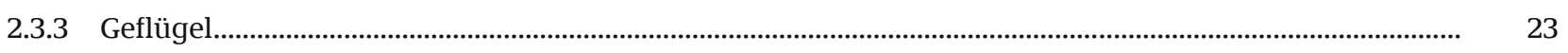

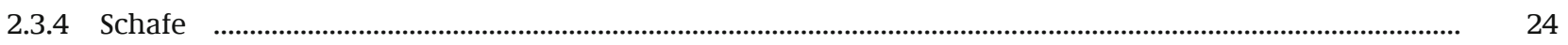

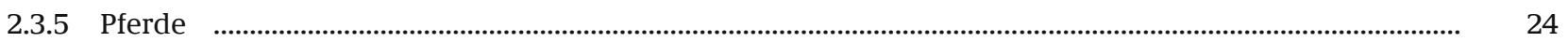

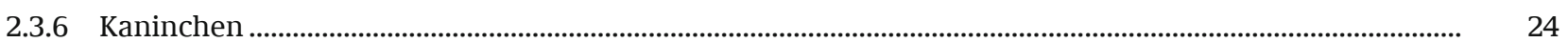

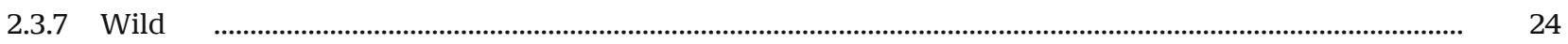

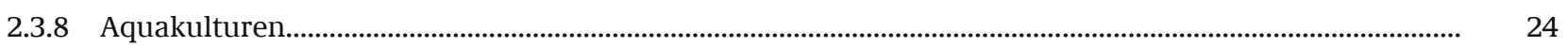

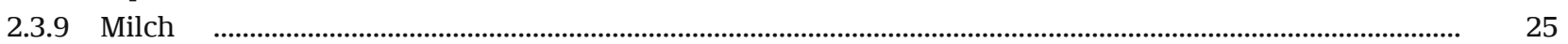

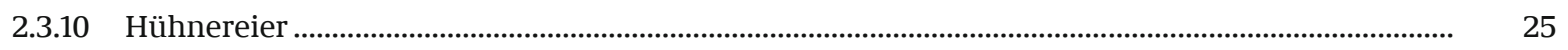

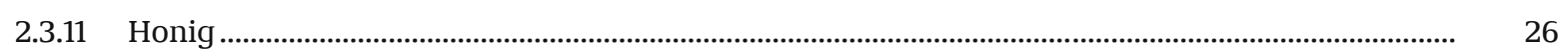

2.4. Bewertungsbericht des BfR zu den Ergebnissen des NRKP 2005 ............................................................................... 26

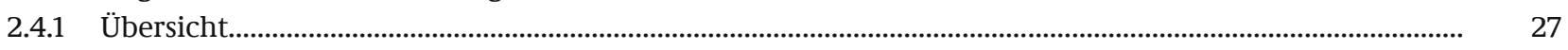

2.4.2 Bewertung der Einzelstoffe ........................................................................................................................... 27

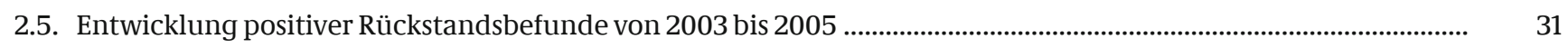

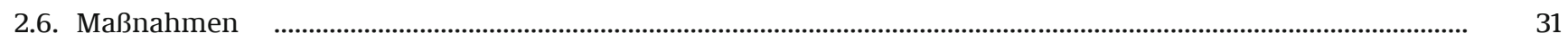

2.6.1 Ermittlung der Ursachen von positiven Rückstandsbefunden ...........................................................................

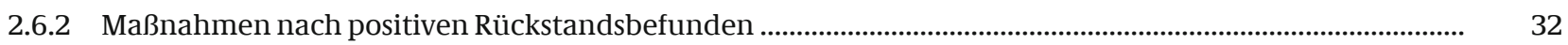


2.7. Zuständige Ministerien.

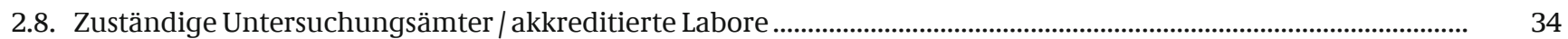

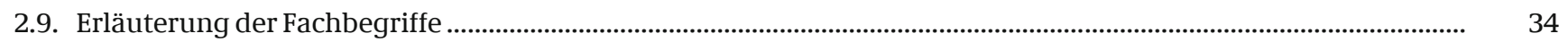

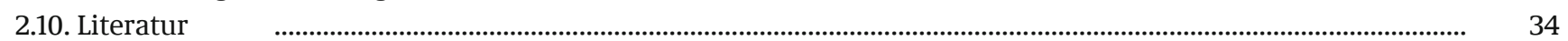

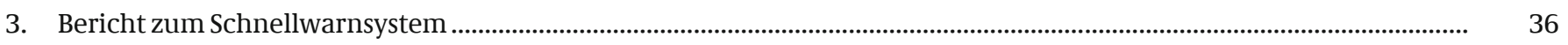

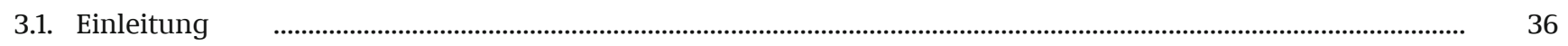

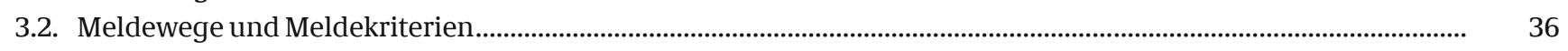

3.2.1 Meldungen aus Deutschland an die Europäische Kommission (,Upstream“-Verfahren)............................... 36

3.2.2 Meldungen von der Europäischen Kommission an die Mitgliedstaaten der EU 37

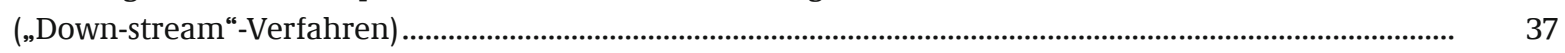

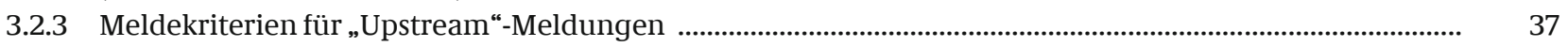

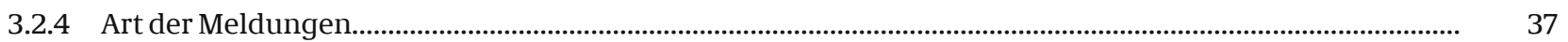

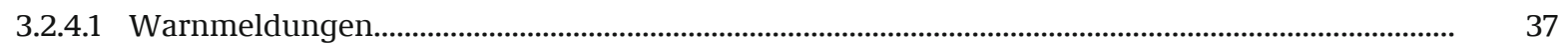

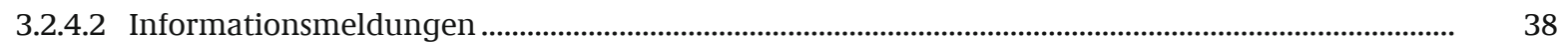

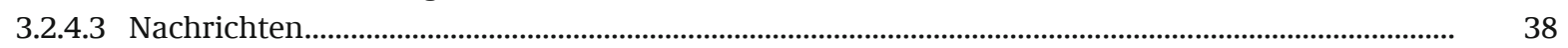

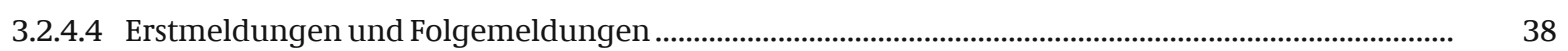

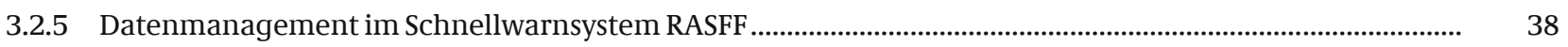

3.2.6 Wöchentliche Berichte der EU-Kommission zum Schnellwarnsystem RASFF ................................................ 38

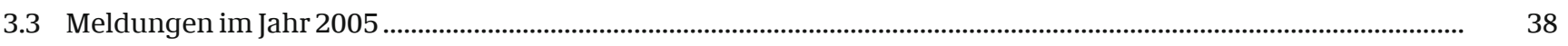

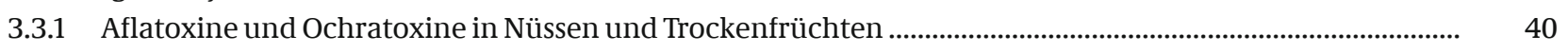

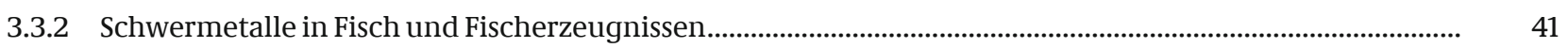

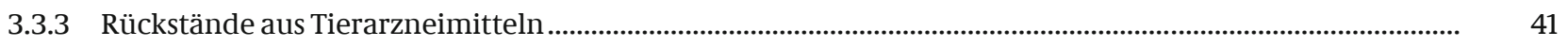

3.3.4 Nicht zugelassene Farbstoffe in Gew

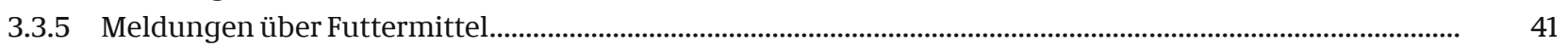

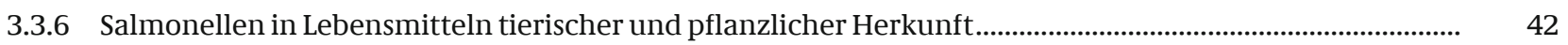

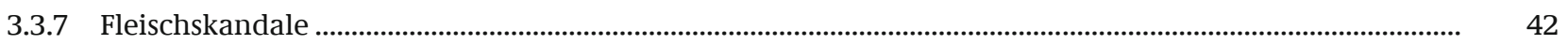

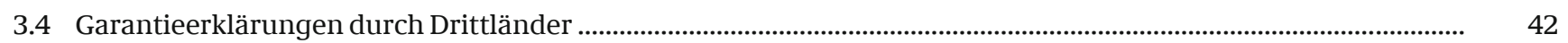

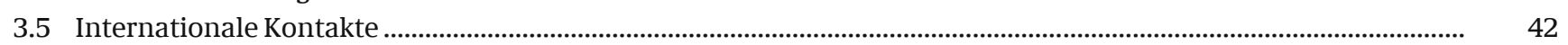

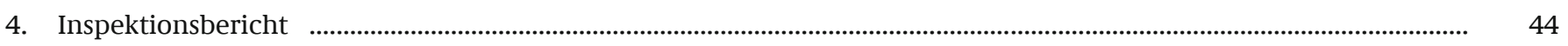

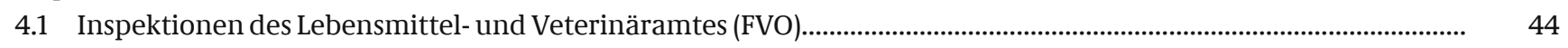

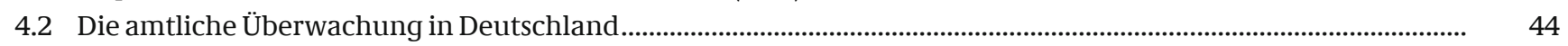

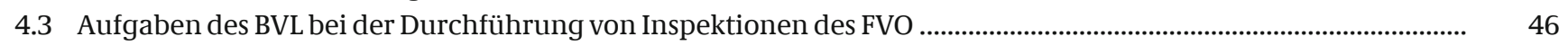

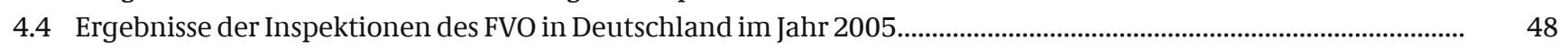

4.4.1 GD SANCO 7528/2005: Bewertung der Durchführung und der Kontrollen hinsichtlich der

EU-Vorschriften über Tiergesundheit beim innergemeinschaftlichen Handel mit Samen und

Embryonen von Hausrindern ....

4.4.2 GD SANCO 7510/2005: Bewertung der Kontrollen von Rückständen und Kontaminanten in lebenden Tieren und tierischen Erzeugnissen einschließlich der Kontrollen von Tierarzneimitteln ............. 49

4.4.3 GD SANCO 7642/2005: Einfuhrkontrollen und Grenzkontrollstellen...

4.4.4 GD SANCO 7643/2005: Illegale Einfuhren von Erzeugnissen tierischen Ursprungs und von 1 ebenden Tieren.

4.4.5 GD SANCO 7583/2005: Bewertung der Hygieneverhältnisse bei der Erzeugung lebender Muscheln und bestimmter Hygienevorschriften in Bezug auf Fischereierzeugnisse, die in der Europäischen

Union in Verkehr gebracht werden

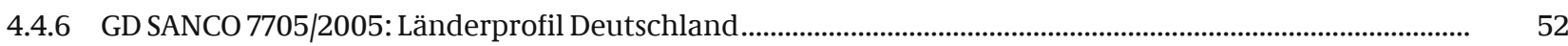

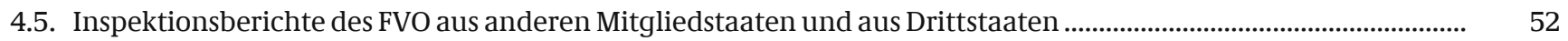

4.6. Fragebögen des FVO.

4.7. Inspektionen von Drittstaaten in Deutschland 